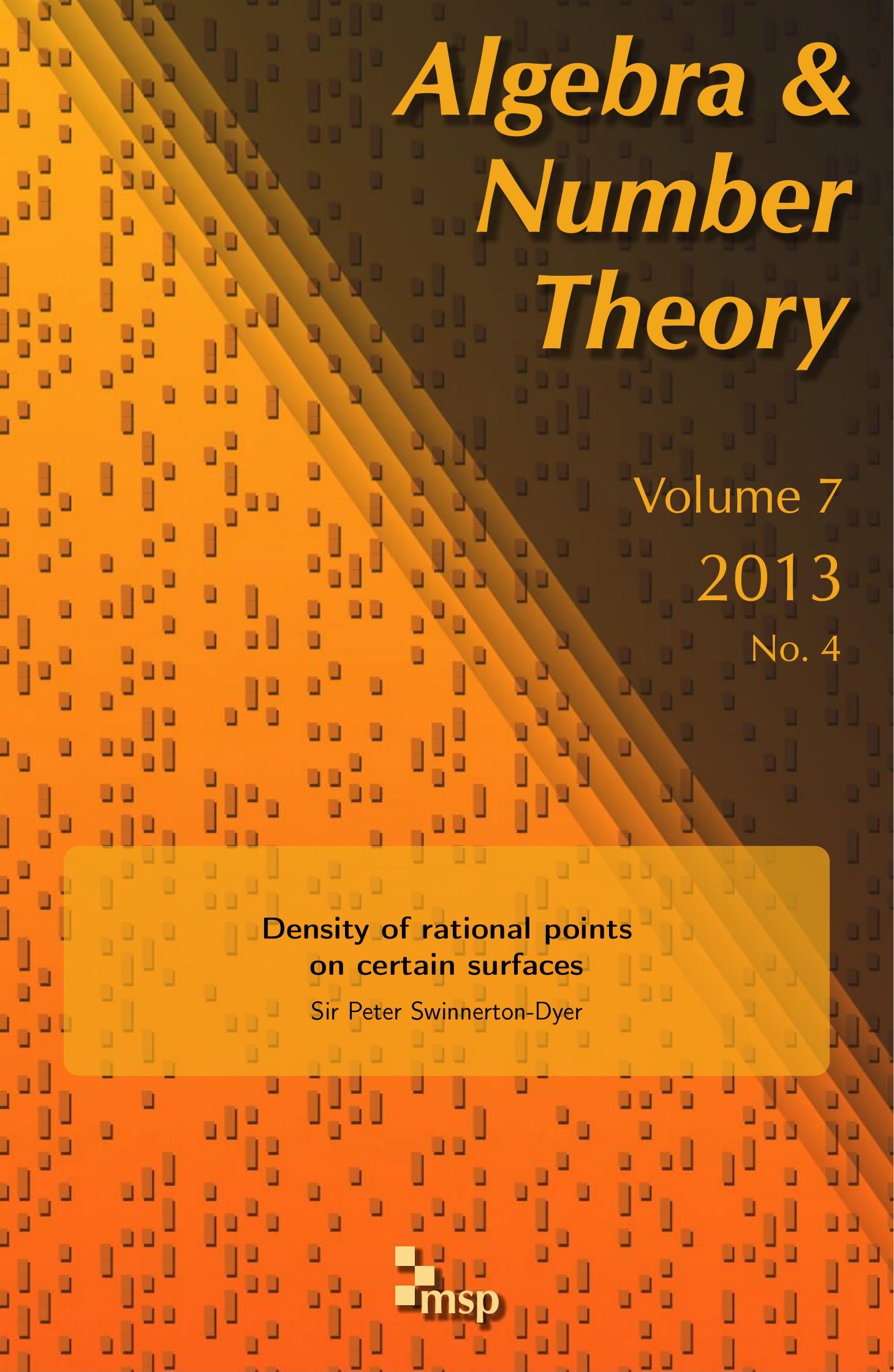




\title{
Density of rational points on certain surfaces
}

\author{
Sir Peter Swinnerton-Dyer
}

Let $V$ be a nonsingular projective surface defined over $\mathbb{Q}$ and having at least two elliptic fibrations defined over $\mathbb{Q}$; the most interesting case, though not the only one, is when $V$ is a $\mathrm{K} 3$ surface with these properties. We also assume that $V(\mathbb{Q})$ is not empty. The object of this paper is to prove, under a weak hypothesis, the Zariski density of $V(\mathbb{Q})$ and to study the closure of $V(\mathbb{Q})$ under the real and the $p$-adic topologies. The first object is achieved by the following theorem:

Let $V$ be a nonsingular surface defined over $\mathbb{Q}$ and having at least two distinct elliptic fibrations. There is an explicitly computable Zariski closed proper subset $X$ of $V$ defined over $\mathbb{Q}$ such that if there is a point $P_{0}$ of $V(\mathbb{Q})$ not in $X$ then $V(\mathbb{Q})$ is Zariski dense in $V$.

The methods employed to study the closure of $V(\mathbb{Q})$ in the real or $p$-adic topology demand an almost complete knowledge of $V$; a typical example of what they can achieve is as follows. Let $V_{c}$ be

$$
V_{c}: X_{0}^{4}+c X_{1}^{4}=X_{2}^{4}+c X_{3}^{4} \quad \text { for } c=2,4 \text { or } 8 ;
$$

then $V_{c}(\mathbb{Q})$ is dense in $V_{c}\left(\mathbb{Q}_{2}\right)$ for $c=2,4,8$.

1. Introduction. Let $V$ be a nonsingular projective surface defined over $\mathbb{Q}$ and having at least two elliptic fibrations defined over $\mathbb{Q}$; the most interesting case, though not the only one, is when $V$ is a $\mathrm{K} 3$ surface with these properties. (By an elliptic fibration we mean a fibration by curves of genus 1 ; we do not assume that these curves have distinguished points, so they need not be elliptic curves in the number-theoretic sense.) We also assume that $V(\mathbb{Q})$ is not empty. The object of this paper is to prove, under a weak hypothesis, the Zariski density of $V(\mathbb{Q})$ and to study the closure of $V(\mathbb{Q})$ under the real and the $p$-adic topologies. These results can be found in Section 2; Sections 3 and 4 contain applications and examples. Note that the geometric terminology in this paper is that of Weil.

For the real and Zariski topologies, such results have been proved for a particular family of such surfaces in [Logan et al. 2010], and for one such surface already in [Swinnerton-Dyer 1968]; see Section 3. I am indebted to the referee for drawing

MSC2010: 11G35.

Keywords: rational points, K3 surfaces. 
my attention to [Bogomolov and Tschinkel 1998], where Proposition 2.13 proves that such $V$ have the closely related property of potential density, and to René Pannekoek for pointing out a major error in an earlier draft.

2. The main theorems. Let $\mathscr{F}$ denote an elliptic fibration of $V$ defined over $\mathbb{Q}$. Then $\mathscr{F}$ is given by a map $\theta: V \rightarrow \mathbb{P}^{1}$ defined over $\mathbb{Q}$ whose generic fibre $\theta^{-1}(t)$ is a curve of genus 1 defined over $\mathbb{Q}(t)$. The Jacobian of $\theta^{-1}(t)$ is also defined over $\mathbb{Q}(t)$. Let $P$ be generic over $\mathbb{Q}(t)$ on this Jacobian and let $W$ be the locus of $P$ over $\mathbb{Q}$; then $P \mapsto t$ gives a natural map $\phi: W \rightarrow \mathbb{P}^{1}$ whose fibre over $t$ is the Jacobian of $\theta^{-1}(t)$, and for any $c$ such that $C=\theta^{-1}(c)$ is nonsingular the Jacobian of $C$ is $J=\phi^{-1}(c)$. In other words, $\phi$ is the Jacobian elliptic fibration associated to $\theta$.

In this situation $C$ is an $n$-covering of $J$ for some $n$, and by abuse of language we can claim that $n$ only depends on the fibration and not on $c$. (The abuse of language here is that $n$ is not unique.) However, this property by itself does not determine the covering map uniquely. We shall require that the covering map is the restriction to $C$ of a rational map $\psi: V \rightarrow W$ defined over $\mathbb{Q}$. One way of ensuring this is as follows. Let $P$ be generic on $V$, let $C$ be the fibre through $P$ and $J$ the corresponding fibre of $W$, let $\mathfrak{a}$ be the section of $C$ by a hyperplane $\Pi$ defined over $\mathbb{Q}$, and write $n=\operatorname{deg}(\mathfrak{a})$. The divisor $n P-\mathfrak{a}$ on $C$ has degree 0 and is defined over $\mathbb{Q}(P)$; so, it is represented by a point $R$ on $J$, also defined over $\mathbb{Q}(P)$, and we can take $\psi$ to be the map defined over $\mathbb{Q}$ that sends $P$ to $R$. This $\psi$ is defined except possibly at points of the singular fibres.

Theorem 1. Let $V$ be a nonsingular surface defined over $\mathbb{Q}$ and having at least two distinct elliptic fibrations. There is an explicitly computable Zariski closed proper subset $X$ of $V$ defined over $\mathbb{Q}$ such that if there is a point $P_{0}$ of $V(\mathbb{Q})$ not in $X$ then $V(\mathbb{Q})$ is Zariski dense in $V$.

Remarks. Here explicitly computable means that if we are given equations for $V$ and the two fibrations $\theta_{1}$ and $\theta_{2}$ we can compute equations for $X$. If $P_{0}$ in $V(\mathbb{Q})$ is known, then in fact it is easy to test whether $P_{0}$ is in $X$ without needing to calculate $X$. A similar theorem holds over any algebraic number field. For applications in this more general case, it will often be helpful to use the sufficient condition due to Lutz for a point on an elliptic curve not to be torsion; see [Silverman 1986, Corollary VIII.7.2].

Proof. Denote by $\mathscr{F}_{v}$ for $v=1,2$ the two given elliptic fibrations on $V$. We retain the notation introduced above, except that objects associated with $\mathscr{F}_{v}$ will have $v$ as a subscript. We take $\mu=3-v$, so that $\{\mu, \nu\}=\{1,2\}$.

We start by defining a certain Zariski closed set $X_{v}$ on $V$. Let $Y_{v}^{\prime}$ be the union of all the singular fibres of $\phi_{\nu}$. By a theorem of Mazur, if $\Gamma$ is an elliptic curve defined over $\mathbb{Q}$ and $R$, also defined over $\mathbb{Q}$, is an $m$-torsion point of $\Gamma$, then $m \leq 10$ 
or $m=12$. If $t$ is generic on $\mathbb{P}^{1}$ with $C_{v}=\theta_{v}^{-1}(t)$ and $J_{v}=\phi_{v}^{-1}(t)$, let $\mathfrak{b}_{v}$ be the union of the $m$-division points on $J_{v}$ for these values of $m$. Thus $\mathfrak{b}_{v}$ is a positive divisor defined over $\mathbb{Q}(t)$, and there is a (reducible) curve $Y_{v}^{\prime \prime}$ on $W_{v}$ defined over $\mathbb{Q}$ and such that the intersection of $Y_{v}^{\prime \prime}$ with $J_{v}$ is $\mathfrak{b}_{v}$. Now let $X_{v}$ be the union of the support of $\psi_{v}^{-1}\left(Y_{v}^{\prime} \cup Y_{\nu}^{\prime \prime}\right)$ and those fibres of $\mathscr{F}_{v}$ that lie entirely in $\psi_{\mu}^{-1}\left(Y_{\mu}^{\prime \prime}\right)$. Clearly $X_{v}$ is Zariski closed. Write $X=X_{1} \cap X_{2}$. The effect of this construction is that if $P_{0}$ is a point of $V(\mathbb{Q})$ not in $X_{v}$ then the fibre $C_{0 v}$ through $P_{0}$ is nonsingular and not in $\psi_{\mu}^{-1}\left(Y_{\mu}^{\prime \prime}\right)$, and $\psi_{\nu}\left(P_{0}\right)$ is not a torsion point of the corresponding $J_{0 v}$. In applications we can replace $X$ by the Zariski closure of $X(\mathbb{Q})$, which will usually be much smaller; in practice one would normally choose $P_{0}$ in $V(\mathbb{Q})$ and then verify that it has the properties stated in the previous sentence for at least one value of $v$.

Now let $P_{0}$ be a point of $V$ not in $X$; without loss of generality we can assume that $P_{0}$ is not in $X_{1}$. For any integer $r$, denote by $P_{r}$ the point obtained from $P_{0}$ by translation along $C_{01}$ by $[r] \psi_{1}\left(P_{0}\right)$, where $[r]$ denotes multiplication by $r$ on $J_{01}$. By construction, the points $P_{r}$ are distinct and defined over $\mathbb{Q}$, so they are Zariski dense in $C_{01}$. Moreover $C_{01}$ is not contained in $\psi_{2}^{-1}\left(Y_{2}^{\prime \prime}\right)$ by definition, and it is not in $\psi_{2}^{-1}\left(Y_{2}^{\prime}\right)$ because a nonsingular fibre of $\mathscr{F}_{1}$ cannot lie in a fibre of $\mathscr{F}_{2}$, so only finitely many of the $P_{r}$ lie in $X_{2}$. Let $C_{r 2}$ be the fibre of $\mathscr{F}_{2}$ through $P_{r}$. The number of $P_{r}$ that lie on a given fibre of $\mathscr{F}_{2}$ is bounded, so there are infinitely many $C_{r 2}$. By an argument like that above for $C_{01}$, if $P_{r}$ is not in $X_{2}$ then $C_{r 2}(\mathbb{Q})$ is Zariski dense in $C_{r 2}$. Hence $V(\mathbb{Q})$, which contains all the $C_{r 2}(\mathbb{Q})$, is Zariski dense in $V$.

For use in the proof of Theorem 2 below, we note that $J_{01}(\mathbb{Q})$ is not merely Zariski dense in $J_{01}$ but dense in the real topology in that connected component of $J_{01}(\mathbb{R})$ which contains the identity element. Hence $C_{01}(\mathbb{Q})$ is not merely Zariski dense in $C_{01}$ but dense in the real topology in that connected component of $C_{01}(\mathbb{R})$ which contains $P_{0}$. A similar remark applies to each $C_{r 2}$ for which $P_{r}$ is not in $X_{2}$. Now let $Z$ be the union of the singular points of the singular fibres of $\mathscr{F}_{1}$ and $\mathscr{F}_{2}$ and the other points at which the fibres of $\mathscr{F}_{1}$ and $\mathscr{F}_{2}$ have intersection multiplicity greater than 1 ; clearly $Z$ is Zariski closed. Let $X$ be as in Theorem 1 and write $U=V \backslash X$.

Theorem 2. Let $\mathscr{R}$ denote the closure of $U(\mathbb{Q})$ in $V(\mathbb{R})$ under the real topology. Then the boundary of $\mathscr{R}$ is contained in $Z \cup X$.

Proof. Let $A$ be a point of $\mathscr{R}$ that is not in $Z \cup X$. The fibres of $\mathscr{F}_{1}$ and $\mathscr{F}_{2}$ through $A$ are nonsingular at $A$ and transversal there; by continuity there is a neighbourhood of $A$ in $V(\mathbb{R})$ at every point of which the same properties hold. After contracting this neighbourhood if necessary, we can suppose that the map $\chi$ defined by $P \mapsto\left(\theta_{1}(P), \theta_{2}(P)\right)$ is a homeomorphism of this neighbourhood to an 
open subset of $\mathbb{P}^{1} \times \mathbb{P}^{1}$ and that the neighbourhood does not meet $X$; without loss of generality we can suppose that it does not meet $X_{1}$. Choose open intervals $I_{1}, I_{2}$ of $\mathbb{P}^{1}$ such that this open subset contains $I_{1} \times I_{2}$ and $I_{1} \times I_{2}$ contains $\chi(A)$. Since $A$ is in $\mathscr{R}$, there is a point $P_{0}$ of $U(\mathbb{Q})$ in $\mathcal{N}=\chi^{-1}\left(I_{1} \times I_{2}\right)$, and $P_{0}$ is not in $X_{1}$. But if $C_{01}$ is the fibre of $\mathscr{F}_{1}$ through $P_{0}$ then $C_{01} \cap \mathcal{N}$ is homeomorphic to $I_{2}$ and hence is connected. By the remark that follows the proof of Theorem 1, $C_{01}(\mathbb{Q})$ is dense in $C_{01} \cap \mathcal{N}$. If $P^{\prime}$ is any point of $C_{01}(\mathbb{Q}) \cap \mathcal{N}$ other than the finitely many which lie in $X_{2}$, and if $C_{2}^{\prime}$ is the fibre of $\mathscr{F}_{2}$ that passes through $P^{\prime}$, then by the same remark $C_{2}^{\prime}(\mathbb{Q}) \cap \mathcal{N}$ is dense in $C_{2}^{\prime} \cap \mathcal{N}$. Hence $V(\mathbb{Q}) \cap \mathcal{N}$ is dense in $\mathcal{N}$, and so is $U(\mathbb{Q}) \cap \mathcal{N}$ since $X$ is nowhere dense in $\mathcal{N}$. In other words, $\mathscr{R} \supset \mathcal{N}$, and therefore $A$ is not in the boundary of $\mathscr{R}$.

The same ideas can be applied to the $p$-adic topologies, though the results that they yield are weaker and the calculations needed to make use of them are more complicated. Let $\mathscr{S}$ be a finite nonempty set of finite primes and let $\mathscr{S}^{+}$be the union of $\mathscr{Y}$ and the infinite place of $\mathbb{Q}$. For the rest of this section we work in the topology associated with $\mathscr{S}$ or $\mathscr{S}^{+}$; unfortunately I have not been able to prove any density theorems for the adelic topology.

Lemma 1. Let

$$
E: \eta^{2}+a_{1} \xi \eta+a_{3} \eta=\xi^{3}+a_{2} \xi^{2}+a_{4} \xi+a_{6}
$$

be an elliptic curve defined over $\mathbb{Q}_{p}$, with all the $a_{i}$ in $p \mathbb{Z}_{p}$, and suppose further that $4 \mid a_{1}$ and $4 \mid a_{3}$ if $p=2$, and that $9 \mid a_{2}$ if $p=3$. Let $\mathbb{Y}_{p}$ be $\mathbb{Z}_{p}$ if $p=2$ or 3 and $p \mathbb{Z}_{p}$ if $p>3$, and write $\zeta=\xi / \eta$. Let $E^{\prime}$ be the union of the point at infinity and the subset of $E\left(\mathbb{Q}_{p}\right)$ for which $\xi^{-1}$ is in $\bigvee_{p}$. Then $E^{\prime}$ is a subgroup of $E\left(\mathbb{Q}_{p}\right)$. Moreover the map

$$
E^{\prime} \rightarrow\left\{\zeta \text { in } \mho_{p}\right\}
$$

is one-one and bicontinuous. If $A_{0}=\left(\xi_{0}, \eta_{0}\right)$ is a point of $E^{\prime}(\mathbb{Q})$ other than the point at infinity, then $E^{\prime}(\mathbb{Q})$ is dense in that part of $E^{\prime}\left(\mathbb{Q}_{p}\right)$ in which $v_{p}(\xi) \leq v_{p}\left(\xi\left(A_{0}\right)\right)$.

Remark. A less ugly proof can be given by using the theory of formal groups; compare [Silverman 1986, Chapter IV]. The lemma is a weaker and easier version of a result due to René Pannekoek [2012]. I am indebted to him for communicating his result to me.

Proof. We can reduce to the case $a_{1}=a_{2}=a_{3}=0$ by an obvious linear transformation, which does not change the description of $E^{\prime}$. If $(\xi, \eta)$ is in $E^{\prime}$ then

$$
3 v(\xi)=2 v(\eta) \leq 0 \text { and therefore } v(\zeta)=v(\xi / \eta)=-\frac{1}{2} v(\xi) \geq 0 .
$$

The map (1) is defined and continuous. Conversely, if we write $u=\xi^{-1}$ then

$$
u=\zeta^{2}\left(1+a_{4} u^{2}+a_{6} u^{3}\right) .
$$


For $\zeta$ in $\mathbb{Y}_{p}$ this has just one solution $u$ in $\mathbb{Y}_{p}$, which is a continuous function of $\zeta$ with $v(u)=2 v(\zeta)$. Hence, the map (1) is a homeomorphism. If $\tilde{E}$ is the reduction of $E \bmod p$, then the image of $E^{\prime}$ is $\tilde{E}\left(\mathbb{F}_{p}\right) \backslash(0,0)$ if $p=2$ or 3 and the point at infinity otherwise. This is a group, so the sum or difference of two points of $E^{\prime}$ is again a point of $E^{\prime}$. Hence $E^{\prime}$ is a subgroup of $E$.

$\mathbb{Z}$ acts on $E^{\prime}$ by $P \mapsto[m] P$. We now show that this map is continuous, so that $\mathbb{Z}_{p}$ acts continuously on $E^{\prime}$. Let $P_{i}=\left(\xi_{i}, \eta_{i}\right)$ for $i=1,2$ be points of $E^{\prime}$ and write $\zeta_{i}=\xi_{i} / \eta_{i}$ and $n_{i}=v\left(\zeta_{i}\right)$. The addition formula on $E$ gives

$$
\xi\left(P_{1}+P_{2}\right)=\alpha^{2}-\xi_{1}-\xi_{2}, \quad \eta\left(P_{1}+P_{2}\right)=-\alpha\left(\xi\left(P_{1}+P_{2}\right)-\xi_{1}\right)-\eta_{1},
$$

where in general $\alpha=\left(\eta_{1}-\eta_{2}\right) /\left(\xi_{1}-\xi_{2}\right)$; if $P_{1}=P_{2}$, then $\alpha=\left(3 \xi_{1}^{2}+a_{4}\right) / 2 \eta_{1}$.

If $p=2$ and $P_{1}=P_{2}$ then $v(\alpha)=-n_{1}-1$ so that $\zeta\left([2] P_{1}\right) \equiv 2 \zeta_{1} \bmod 2^{n_{1}+2}$. If $p=3$ and $P_{1}=P_{2}$ then $v(\alpha) \geq 1-n_{1}$, so that $\xi\left([2] P_{1}\right) \equiv-2 \xi_{1} \bmod 3^{2-2 n_{1}}$ and $\eta\left([2] P_{1}\right) \equiv-\eta_{1} \bmod 3^{2-3 n_{1}}$; thus $\zeta\left([2] P_{1}\right) \equiv 2 \zeta_{1} \bmod 3^{2+n_{1}}$. If instead $P_{2}=[2] P_{1}$ then $\alpha \equiv 2 \eta_{1} / 3 \xi_{1} \bmod 3^{-n_{1}}$, so that $\xi\left([3] P_{1}\right) \equiv \alpha^{2} \bmod 3^{-2 n_{1}}$ and $\zeta\left([3] P_{1}\right) \equiv 3 \zeta_{1}$ $\bmod 3^{2+n_{1}}$. If $p>3$ then $\xi_{1} \equiv \zeta_{1}^{-2} \bmod p^{2-2 n_{1}}$ and $\eta_{1} \equiv \zeta_{1}^{-3} \bmod p^{2-3 n_{1}}$. If $P_{1}=P_{2}$ then $\alpha \equiv \frac{3}{2} \zeta_{1}^{-1} \bmod p^{2-n_{1}}$ so that $\xi\left([2] P_{1}\right) \equiv \frac{1}{4} \zeta_{1}^{-2} \bmod p^{2-2 n_{1}}$ and $\eta\left([2] P_{1}\right) \equiv \frac{1}{8} \zeta_{1}^{-3} \bmod p^{2-3 n_{1}}$; thus $\zeta\left([2] P_{1}\right) \equiv 2 \zeta_{1} \bmod p^{2+n_{1}}$. For $3 \leq m \leq p$ we now take $P_{2}=[m-1] P_{1}$; it follows by induction on $m$ that (as $p$-adic numbers)

$$
\begin{aligned}
\alpha\left(m^{2}-m\right) & \equiv\left(m^{2}-m+1\right) \zeta_{1}^{-1} \bmod m p^{2-n_{1}}, \\
m^{2} \xi\left([m] P_{1}\right) & \equiv \zeta_{1}^{-2} \bmod m^{2} p^{2-2 n_{1}}, \\
m^{3} \eta\left([m] P_{1}\right) & \equiv \zeta_{1}^{-3} \bmod m^{3} p^{2-3 n_{1}},
\end{aligned}
$$

and therefore $\zeta\left([m] P_{1}\right) \equiv m \zeta_{1} \bmod m p^{2+n_{1}}$.

It follows by induction on $r$ that $\zeta\left(\left[p^{r}\right] P_{1}\right) \equiv p^{r} \zeta_{1} \bmod p^{r+n_{1}+1}$ for any $p$ and any $r>0$. Now suppose that $n_{2}>n_{1}$. By (2),

$$
\xi_{2} \equiv \zeta_{2}^{-2} \bmod p^{2 n_{2}+2}, \quad \eta_{2} \equiv \zeta_{2}^{-3} \bmod p^{3 n_{2}+2}
$$

and therefore $\alpha \equiv \zeta_{2}^{-1}\left(1-\eta_{1} / \eta_{2}+\xi_{1} / \xi_{2}\right) \bmod p^{3 n_{2}-4 n_{1}}$. Hence (as $p$-adic numbers)

$$
\xi\left(P_{1}+P_{2}\right) \equiv \xi_{1}-2 \eta_{1} \zeta_{2} \bmod 2 p^{2 n_{2}-4 n_{1}}
$$

by the first equation of (3). Now $\eta\left(P_{1}+P_{2}\right) \equiv \eta_{1} \bmod p^{-n_{2}-2 n_{1}}$ by the second equation of (3). This tells us which square root to take in the equation for $E$, and combining this fact with (4) gives

$$
\eta\left(P_{1}+P_{2}\right) \equiv \eta_{1} \bmod 2 p^{n_{2}-4 n_{1}} .
$$

Now (4) and (5), taken with the first sentence of this paragraph, prove that $\mathbb{Z}$ acts continuously on $E^{\prime}$. Hence $\mathbb{Z}_{p}$ acts on $E^{\prime}$ too, and this action is continuous. If $A_{0}$ is as in the lemma and $A$ satisfies $v(\xi) \leq v\left(\xi\left(A_{0}\right)\right)$, a successive approximation 
argument shows that there is an $m$ in $\mathbb{Z}_{p}$ such that $A=[m] A_{0}$. If $n$ in $\mathbb{Z}$ is close to $m$ then $[n] A_{0}$ will be close to $A$.

Theorem 3. Let $X_{1}, X_{2}$ be as in the proof of Theorem 1, and let $P_{0}$ be a point of $V(\mathbb{Q})$ not in $X_{1} \cup X_{2}$. Then there is a constructable open neighbourhood $\mathscr{D}$ of $P_{0}$ in $\prod V\left(\mathbb{Q}_{p}\right)$, where the product is taken over all $p$ in $\mathscr{T}$, such that $V(\mathbb{Q})$ is dense in $\mathscr{D}$. A similar result holds for $\mathscr{S}^{+}$.

Proof. In the notation of Lemma 1, we can rescale the equations of $W$ so that $J_{01}$ satisfies the conditions imposed on $E$ for each $p$ in $\mathscr{Y}$, where $J_{01}$ is again the Jacobian of the fibre of $\mathscr{F}_{1}$ through $P_{0}$. For each such $p$, let $r_{p}$ be the least positive integer $r$ such that $[r] \psi_{1}\left(P_{0}\right)$ is in the set $J_{01}^{\prime}$ derived from $J_{01}$ in the way that $E^{\prime}$ was derived from $E$ and let $r^{*}$ be a convenient common multiple of the $r_{p}$ for $p$ in $\mathscr{Y}$. Let $\left(\xi^{*}, \eta^{*}\right)=\left[r^{*}\right] \psi_{1}\left(P_{0}\right)$ and let $m_{p}$ be the exact power of $p$ that divides $\xi^{*} / \eta^{*}$. Let $\mu_{p 1}\left(P_{0}\right) \subset J_{01}\left(\mathbb{Q}_{p}\right)$ be the subset of $J_{01}^{\prime}$ which corresponds to the $\zeta$ in $m_{p} \mathbb{Z}_{p}$ under the isomorphism corresponding to (1), and let $\mathcal{N}_{p 1}\left(P_{0}\right) \subset C_{01}\left(\mathbb{Q}_{p}\right)$ consist of the points obtained from $P_{0}$ by translation by an element of $\mu_{p 1}\left(P_{0}\right)$. If we denote by $P_{r}$ the translation of $P_{0}$ by $[r] \psi_{1}\left(P_{0}\right)$ then by the Chinese remainder theorem the $P_{r}$ for which $r$ is a multiple of $r^{*}$ are distinct and dense in $\prod \mathcal{N}_{p 1}\left(P_{0}\right)$ where the product is taken over all $p$ in $\mathscr{S}$.

We can apply a similar construction with $v=2$ to obtain a neighbourhood $\mathscr{A}$ of $P_{0}$ in $\prod C_{02}\left(\mathbb{Q}_{p}\right)$ in which points of $V(\mathbb{Q})$ are dense. If in the construction of the previous paragraph we replace $P_{0}$ by any point $P^{\prime}$ in $\mathscr{A}$, everything involved in the construction varies continuously with $P^{\prime}$; so after reducing $\mathscr{A}$ if necessary, we can assume that the $r_{p}$ and the $m_{p}$ are independent of $\mathscr{A}$. One consequence of this is that if $P^{\prime}=\prod P_{p}^{\prime}$ then $\psi_{1}\left(P_{p}^{\prime}\right)$ cannot be a torsion point; and it is now easy to see that we can choose $\mathscr{D}$ to be the union of the $\prod \mathcal{N}_{p 1}\left(P^{\prime}\right)$ where the union is taken over all points $P^{\prime}$ of $\mathscr{A}$.

The corresponding result for $\mathscr{S}^{+}$now follows easily. For let $\mathscr{D}_{0}$, containing $P_{0}$, be a small open subset of the $\mathscr{D}$ associated with $\mathscr{Y}$; by diminishing $\mathscr{D}_{0}$ we can further assume that it is disjoint from $X_{1} \cup X_{2}$. Let $\mathscr{R}\left(\mathscr{D}_{0}\right)$ be the closure of the projection of $\phi V(\mathbb{Q}) \cap\left(\mathscr{D}_{0} \times V(\mathbb{R})\right)$ onto $V(\mathbb{R})$, where $\phi$ is the obvious diagonal map. Then it is enough to show that there is a nonempty open set contained in $\mathscr{R}\left(\mathscr{D}_{0}\right)$ and independent of $\mathscr{D}_{0}$. Suppose that $r^{*}$ is even and divisible by a high enough power of every $p$ in $\mathscr{S}$ and by all the $r_{p}$ defined in the second paragraph of this proof. Then $r^{*}$ depends only on $\mathscr{D}_{0}$, and the translation of $P_{0}$ by $\left[r^{*}\right] \psi_{1}\left(P_{0}\right)$ lies in $\mathscr{D}_{0}$ and is dense in the connected component containing $P_{0}$ of the fibre of $\mathscr{F}_{1}$ through $P_{0}$. There is a corresponding result for $\mathscr{F}_{2}$. So the argument used to prove Theorem 2 still applies in this situation, and the boundary of $\mathscr{R}\left(\mathscr{D}_{0}\right)$ lies in $Z \cup X$, which is independent of $\mathscr{D}_{0}$. Thus $\mathscr{R}\left(\mathscr{D}_{0}\right)$ contains that connected component of $V(\mathbb{R}) \backslash(Z \cup X)$ in which $P_{0}$ lies. 
In what circumstances will the machinery of Theorem 3 enable us to prove that $\overline{V(\mathbb{Q})}$, in the topology generated by $\mathscr{Y}$, is at least as large as it actually is? (A similar question for $\mathscr{Y}^{+}$can be addressed in much the same way; the details are left to the reader.) For this purpose we make the temporary assumption that $\overline{V(\mathbb{Q})}$ is open as well as closed - which would certainly hold if for example the entire obstruction to weak approximation consists of finitely many Brauer-Manin conditions.

Suppose that $P_{0}$ is in $V\left(\mathbb{Q}_{p}\right)$ and not on a singular fibre of $\mathscr{F}_{1}$. Let $r_{p}$ be the least positive $r$ such that $[r] \psi_{1}\left(P_{0}\right)$ is in $J_{01}^{\prime}$ in the notation of the last proof; then $\psi_{1}\left(P_{0}\right)$ can only be a torsion point of $J_{01}$ if $\left[r_{p}\right] \psi_{1}\left(P_{0}\right)$ is the identity element of $J_{01}$. Thus the points $P_{0}$ in $V\left(\mathbb{Q}_{p}\right)$ not on a singular fibre of $\mathscr{F}_{1}$ and for which $\psi_{1}\left(P_{0}\right)$ is torsion lie on a (reducible) curve $Z_{1 p}$. Let $\mathscr{X}$ be a compact open subset of $\prod V\left(\mathbb{Q}_{p}\right)$ such that if $\prod A_{p}$ is in $\mathscr{X}$ then no $A_{p}$ lies in $Z_{1 p}$ or on a singular fibre of $\mathscr{F}_{1}$. By compactness, the values of $m_{p}$ and $r_{p}$ associated with $A_{p}$ are bounded as $\prod A_{p}$ varies in $\mathscr{X}$; thus if $P_{0}$ is a point of $\mathscr{L} \cap V(\mathbb{Q})$ the size of the domain $\mathscr{D}$ associated with $P_{0}$ in the sense of Theorem 3 is bounded below. This implies that provided $V(\mathbb{Q})$ is actually dense in $\mathscr{L}$ we can prove this by exhibiting finitely many points of $V(\mathbb{Q})$. What we want to prove for any particular $V$ is of course stronger than this. But these considerations do suggest that it will be easiest to prove that $\overline{V(\mathbb{Q})}$ as a subset of $\prod V\left(\mathbb{Q}_{p}\right)$ is as large as it actually is when there are so many elliptic fibrations $\mathscr{F}_{v}$ that $\bigcap X_{v}(\mathbb{Q})$ is empty. Notice that for specific $V$ we can usually modify the argument so as to make $\mathscr{D}$ substantially larger than the $\mathscr{D}$ constructed above. We illustrate this in Section 4.

3. An example of Theorems 1 and 2 . The best known examples of $\mathrm{K} 3$ surfaces with at least two elliptic pencils are given by equations of the form

$$
a_{0} X_{0}^{4}+a_{1} X_{1}^{4}+a_{2} X_{2}^{4}+a_{3} X_{3}^{4}=0 \quad \text { where } a_{0} a_{1} a_{2} a_{3}=b^{2} .
$$

Theorems on the density of rational points in the real and the Zariski topologies on such a surface, assuming that it contains at least one rational point lying outside a certain subvariety, already appear in [Logan et al. 2010]. The earliest example of such a result, for the real topology and one special $V$, appears to be [SwinnertonDyer 1968]. The arguments in [Logan et al. 2010] do not appear to extend to the most general $V$ considered in this paper, but the methods of this paper do yield, in the case of (6), a simpler proof of their result, which is stated here as Theorem 4. For this, and for later applications, we need to calculate the exceptional sets $X$ and $Z$ for (6), and for this it is convenient to repeat some formulae from [Swinnerton-Dyer 2000], though the reader is warned that there are some errors in that paper.

Denote by $V$ the K3 surface given by (6). The union of the singular fibres of $V$ is the union of the 48 lines on $V$. There is an obvious map from $V$ to

$$
T: a_{0} Y_{0}^{2}+a_{1} Y_{1}^{2}+a_{2} Y_{2}^{2}+a_{3} Y_{3}^{2}=0 .
$$


Since $a_{0} a_{1} a_{2} a_{3}$ is a square, each of the two families of lines on $T$ is defined over $\mathbb{Q}$, and the lifts of these families to $V$ give two elliptic fibrations on $V$. The fibre through $P_{0}=\left(x_{0}, x_{1}, x_{2}, x_{3}\right)$ of one of the fibrations is given by four equations of the form

$$
d_{i l} X_{i}^{2}+d_{j l} X_{j}^{2}+d_{k l} X_{k}^{2}=0
$$

where $i, j, k, l$ are $0,1,2,3$ in some order. Any three of these equations are linearly dependent. A possible set of values of the coefficients is

$$
\left.\begin{array}{ll}
d_{01}=b\left(a_{2} x_{2}^{4}+a_{3} x_{3}^{4}\right), & d_{23}=\left(b / a_{0} a_{1}\right) d_{01}, \\
d_{02}=-a_{2}\left(a_{0} a_{3} x_{0}^{2} x_{3}^{2}+b x_{1}^{2} x_{2}^{2}\right), & d_{31}=\left(b / a_{0} a_{2}\right) d_{02}, \\
d_{03}=a_{3}\left(a_{0} a_{2} x_{0}^{2} x_{2}^{2}-b x_{1}^{2} x_{3}^{2}\right), & d_{12}=\left(b / a_{0} a_{3}\right) d_{03},
\end{array}\right\}
$$

together with $d_{j i}=-d_{i j}$ for all $i, j$. The $d_{i j}$ are nonzero since the fibre is absolutely irreducible. In these formulae we can permute the subscripts, provided that for an odd permutation we also change the sign of $b$. The Jacobian of this fibre can be written in the form

$$
\eta^{2}=\left(\xi-c_{1}\right)\left(\xi-c_{2}\right)\left(\xi-c_{3}\right)
$$

where

$$
c_{1}-c_{2}=d_{03} d_{21}, \quad c_{2}-c_{3}=d_{01} d_{32}, \quad c_{3}-c_{1}=d_{02} d_{13} .
$$

If temporarily we write $P=(\xi, \eta)$, then

$$
\xi([2] P)-c_{2}=\frac{\left(\left(\xi-c_{2}\right)^{2}-d_{01} d_{03} d_{21} d_{23}\right)^{2}}{4 \eta^{2}} .
$$

The map $\psi: V \rightarrow W$, which is effectively the canonical map from each fibre to its Jacobian, can be taken to be

$$
\eta=d_{12} d_{23} d_{31} X_{1} X_{2} X_{3} / X_{0}^{3}, \quad \xi-c_{i}=d_{i j} d_{k i} X_{i}^{2} / X_{0}^{2}
$$

where $i, j, k$ is any permutation of $1,2,3$. This map has degree 4 . There is also an identification of the fibre with its Jacobian which maps $P_{0}$ to the identity. This satisfies

$$
\xi-c_{i}=d_{0 j} d_{0 k} \frac{d_{j i} x_{j} X_{j}+d_{k i} x_{k} X_{k}-d_{0 i} x_{0} X_{0}}{d_{i 0} x_{i} X_{i}+d_{j 0} x_{j} X_{j}+d_{k 0} x_{k} X_{k}}
$$

where again $i, j, k$ is any permutation of $1,2,3$. The formula for $\eta$ is too complicated to be useful. But an immediate deduction from the formula for $\xi$ is that the translation along the fibre of either system by the 2-division point $\left(c_{i}, 0\right)$ is obtained by changing the signs of $x_{0}$ and $x_{i}$. We shall use this in the proof of Theorem 4. To obtain formulae for the other fibration we need only replace $b$ by $-b$ in all the formulae of this paragraph. 
Lemma 2. Suppose that the point $A_{0}$ given by (13) with $x_{i}$ for $X_{i}$ is a rational torsion point, that $x_{0} x_{1} x_{2} x_{3} \neq 0$ and that $P_{0}$ does not lie on any of the lines of $V$. Then

$$
4 a_{j}^{2} d_{k l}^{2} x_{j}^{4} x_{k}^{2} x_{l}^{2}+d_{j k} d_{j l}\left(a_{i} x_{i}^{4}+a_{j} x_{j}^{4}\right)^{2}=0
$$

for some permutation $i, j, k, l$ of $0,1,2,3$. This condition depends only on the value of $i$.

Proof. The point $A_{i}$ that is the translation of $A_{0}$ by the 2-division point $\left(c_{i}, 0\right)$ on the Jacobian satisfies

$$
\xi-c_{i}=\frac{d_{0 j} d_{k 0} x_{0}^{2}}{x_{i}^{2}}, \quad \xi-c_{j}=\frac{d_{0 k} d_{k j} x_{k}^{2}}{x_{i}^{2}}, \quad \xi-c_{k}=\frac{d_{0 j} d_{j k} x_{j}^{2}}{x_{i}^{2}}
$$

where again $i, j, k$ is a permutation of $1,2,3$. We can restore the symmetry by writing

$$
c_{i}=c_{i 0}=c_{0 i}=c_{j k}=c_{k j} ;
$$

thus we have $c_{i k}-c_{j k}=d_{i j} d_{k l}$ for any even permutation $i, j, k, l$ of $0,1,2,3$. The point $A_{i}$ is now given by

$$
\xi-c_{i j}=d_{j k} d_{l j} x_{j}^{2} / x_{i}^{2}
$$

where $i, j, k, l$ is any permutation of $0,1,2,3$; in particular $A_{i}$ is not a 2-division point. Such a value of $\xi$ corresponds to two distinct rational values of $\eta$, so we appear to have constructed twelve torsion points on (10). If these are not all distinct then at least two of the four values of $\xi$ given by (17) must be equal. Suppose for example that the values of $\xi$ given by $i=0$ and $i=1$ are equal; then by comparing the two formulae for $\xi-c_{1}$ we obtain $a_{0} x_{0}^{4}+a_{1} x_{1}^{4}=0$, which implies that $P_{0}$ lies on one of the lines of $V$.

Suppose instead that the twelve torsion points exhibited above are all distinct, so that by Mazur's theorem they form a group of order 12. Without loss of generality we can assume that one of the two 3-division points is $A_{0}$. The condition that $(\xi, \eta)$ is a 3-division point of (10) can be written in the form

$$
4 \eta^{2}\left(\xi-c_{1}\right)=\left\{\left(\xi-c_{1}\right)^{2}-\left(c_{1}-c_{2}\right)\left(c_{1}-c_{3}\right)\right\}^{2},
$$

which reduces by (10) and (11) to

$$
4 a_{1}^{2} d_{23}^{2} x_{1}^{4} x_{2}^{2} x_{3}^{2}+d_{12} d_{13}\left(a_{0} x_{0}^{4}+a_{1} x_{1}^{4}\right)^{2}=0 .
$$

Symmetry now completes the proof of the lemma.

Theorem 4. Suppose that $V$ is given by (6). If there is a rational point $P_{0}=$ $\left(x_{0}, x_{1}, x_{2}, x_{3}\right)$ on $V$ with $x_{0} x_{1} x_{2} x_{3} \neq 0$ and not lying on one of the lines of $V$, then $V(\mathbb{Q})$ is Zariski dense on $V$ and dense in $V(\mathbb{R})$. 
Proof. We retain the notation in the proof of Theorem 1. To deduce Zariski density from Theorem 1 we need to show that $P_{0}$ does not lie in $X$. The first step is to show that the fibre of $\mathscr{F}_{v}$ is not contained in $\psi_{\mu}^{-1}\left(Y_{\mu}^{\prime \prime}\right)$ - in other words, that if $P^{\prime}$ is generic on the fibre of $\mathscr{F}_{\nu}$ through $P_{0}$ then $\psi_{\mu}\left(P^{\prime}\right)$ is not a torsion point. Suppose otherwise; then the exact order of this torsion point cannot depend on the choice of $P^{\prime}$, provided that $P^{\prime}$ does not lie on a singular fibre of $\mathscr{F}_{\mu}$. By the proof of Lemma 2 this exact order must be 3 or 6 . But by changing the signs of a suitable pair of $x_{i}$ we can alter this order from 3 to 6 or from 6 to 3 , and this is a contradiction.

It is now enough to show that $P_{0}$ does not lie in $\left(\psi_{1}^{-1} Y_{1}^{\prime \prime}\right) \cap\left(\psi_{2}^{-1} Y_{2}^{\prime \prime}\right)$; by Lemma 2 this is equivalent to showing that it does not satisfy (15) for both $b$ and $-b$. Suppose otherwise; without loss of generality we can assume that for $b>0$ it satisfies (15) with $i=0$ and that $a_{0}, a_{2}$ are positive and $a_{1}, a_{3}$ negative. In (15) we take $j=1, k=2, l=3$; by (9) we obtain

$$
a_{0} a_{2} a_{3} x_{0}^{4} x_{2}^{2} x_{3}^{2}+a_{2} b x_{0}^{2} x_{1}^{2} x_{2}^{4}-a_{3} b x_{0}^{2} x_{1}^{2} x_{3}^{4}+3 a_{1} a_{2} a_{3} x_{1}^{4} x_{2}^{2} x_{3}^{2}=0 .
$$

On the left the first term is negative and the other three are all positive; so $a_{0} a_{2} x_{0}^{2} x_{2}^{2}>b x_{1}^{2} x_{3}^{2}$ and $a_{0} x_{0}^{4}>-3 a_{1} x_{1}^{4}$. The first of these, taken with (6), implies that $a_{0} x_{0}^{4}$ and $a_{2} x_{2}^{4}$ lie between $-a_{1} x_{1}^{4}$ and $-a_{3} x_{3}^{4}$; so

$$
-a_{3} x_{3}^{4}>\left(a_{0} x_{0}^{4} \text { and } a_{2} x_{2}^{4}\right)>-a_{1} x_{1}^{4} .
$$

We can argue similarly for any one of the four equations (15) with $-b$ for $b$; the only one which yields a condition compatible with (19) is the one with $i=2$. Remembering that we can cyclically permute the subscripts $1,2,3$ in (18), we therefore have

$$
\begin{aligned}
& a_{0} a_{3} a_{1} x_{0}^{4} x_{3}^{2} x_{1}^{2}+a_{3} b x_{0}^{2} x_{2}^{2} x_{3}^{4}-a_{1} b x_{0}^{2} x_{2}^{2} x_{1}^{4}+3 a_{2} a_{3} a_{1} x_{2}^{4} x_{3}^{2} x_{1}^{2}=0 \\
& 3 a_{0} a_{3} a_{1} x_{0}^{4} x_{3}^{2} x_{1}^{2}+a_{3} b x_{0}^{2} x_{2}^{2} x_{3}^{4}-a_{1} b x_{0}^{2} x_{2}^{2} x_{1}^{4}+a_{2} a_{3} a_{1} x_{2}^{4} x_{3}^{2} x_{1}^{2}=0 .
\end{aligned}
$$

The difference of these is $2 a_{1} a_{3} x_{1}^{2} x_{3}^{2}\left(a_{0} x_{0}^{4}-a_{2} x_{2}^{4}\right)=0$. But now either of the last two displayed equations implies that $a_{3} x_{3}^{4} / a_{1} x_{1}^{4}=(2+\sqrt{5})^{2}$, so that $P_{0}$ would not be rational. This completes the proof of Zariski density.

To deduce density in $V(\mathbb{R})$ from Theorem 2 we still need to find the points at which nonsingular fibres of $\mathscr{F}_{1}$ and $\mathscr{F}_{2}$ touch. These fibres are the pull-backs of the lines on (7), on which two lines of opposite systems are always transversal; so the points at which fibres of $\mathscr{F}_{1}$ and $\mathscr{F}_{2}$ touch are just those which have multiplicity greater than 1 in the pull-back of a point of (7). These are just those points of $V$ at which at least one of the $x_{i}$ vanishes. Thus a maximum connected component in $V(\mathbb{R})$ of the complement of $Z$ consists of all the points at which the signs of the $X_{i} / X_{j}$ take assigned values; and since we can vary the signs of the $x_{i}$, if one of 
these connected components meets $V(\mathbb{Q})$ then each of them does. Thus the closure of $V(\mathbb{Q})$ contains the whole of $V(\mathbb{R}) \backslash Z$, and therefore the whole of $V(\mathbb{R})$.

4. An example of Theorem 3. In this section we treat two particular examples of Theorem 3, for each of which $V$ has the form

$$
a_{0} X_{0}^{4}+a_{1} X_{1}^{4}=a_{0} X_{2}^{4}+a_{1} X_{3}^{4} \quad \text { with } a_{0}, a_{1} \text { positive. }
$$

In constructing examples to illustrate the machinery of Theorem 3, we have to choose a suitable $\mathscr{T}$. It is not unreasonable to hope that the only places involved in describing the obstruction to weak approximation on a given $V$ are those at which $V$ has bad reduction and possibly the infinite place. (The corresponding result for nonsingular cubic surfaces is known; see [Swinnerton-Dyer 2001]. An analogous result for the Brauer-Manin obstruction on arbitrary diagonal quartic surfaces defined over $\mathbb{Q}$ is due to Colliot-Thélène and Skorobogatov [2013].) If we want $V$ to have the form (6) and to have bad reduction only at 2, then there are just seven such $V$ which are everywhere locally soluble, and there are just two of them for which the arithmetic part of the Brauer-Manin obstruction is trivial. (See Bright's table [2002, Appendix A].) These can conveniently be written as

$$
V_{c}: X_{0}^{4}+c X_{1}^{4}=X_{2}^{4}+c X_{3}^{4} \quad \text { for } c=a_{1} / a_{0}=2,4 \text { or } 8 .
$$

Here $V_{2}$ and $V_{8}$ are the same, but by considering both we can confine ourselves to the study of points for which the $X_{i}$ are integers with $X_{0}, X_{2}$ odd. In Bright's table $V_{4}$ is case A75, and Ieronymou, Skorobogatov and Zarhin [Ieronymou et al. 2011] have shown that it has no transcendental Brauer-Manin obstruction either. $V_{2}$ and $V_{8}$ are instances of Bright's case A104. We shall prove

Theorem 5. $V_{c}(\mathbb{Q})$ is dense in $V_{c}\left(\mathbb{Q}_{2}\right)$ for $c=2,4,8$.

There is a substantial disparity between the numbers of essentially distinct nontrivial solutions of $V_{2}$ and of $V_{4}$ : for example, $V_{4}$ has 599 such solutions of height less than 25000, the smallest having height 9 , whereas $V_{2}$ has 43 such solutions of height less than 25000 , the smallest having height 139 . This is perhaps accounted for by the fact that the Nèron-Severi group of $V_{4}$ has rank 9 whereas that of $V_{2}$ has rank 6 .

The general surface (20) is case A45 in Bright's table. This surface contains four rational lines. If it has other parametric solutions, which I doubt, even the simplest nonsingular one has degree at least 17. It has three kinds of elliptic pencils of low degree, where the degree means the degree of the general curve of the pencil. Apart from these, which are described below, if there are any other elliptic pencils whose general fibre is nonsingular, they have degree at least 19.

There are four elliptic pencils of degree 3 , the curves of such a pencil being those which lie in a plane through one of the rational lines and are residual to that line. 
A typical such pencil, which we shall call $\mathscr{F}_{3}$, consists of the curves given by

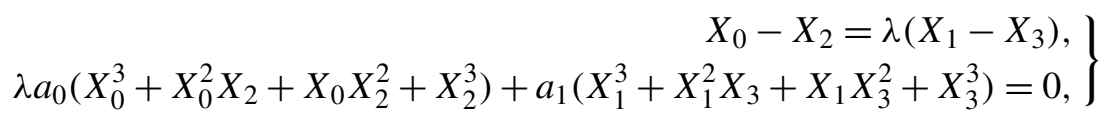

where $\lambda$ is a parameter. This curve contains the rational point $(\lambda, 1,-\lambda,-1)$ and can therefore be taken to be its own Jacobian. In general it has no rational torsion points, but if $\lambda=-a_{1} / a_{0} \alpha^{3}$ then it contains the point $(\alpha, 1, \alpha, 1)$, which is a 2-division point. By means of the transformation

$$
\left.\begin{array}{l}
X_{0}=-a_{1} X+\lambda Y, \quad X_{1}=a_{0} \lambda\left(a_{1}^{2}-a_{0}^{2} \lambda^{8}\right) Z+a_{0} \lambda^{3} X+Y, \\
X_{2}=-a_{1} X-\lambda Y, \quad X_{3}=a_{0} \lambda\left(a_{1}^{2}-a_{0}^{2} \lambda^{8}\right) Z+a_{0} \lambda^{3} X-Y,
\end{array}\right\}
$$

whose inverse is

$$
X=-\frac{X_{0}+X_{2}}{2 a_{1}}, \quad Y=\frac{X_{1}-X_{3}}{2}, \quad Z=\frac{X_{1}+X_{3}-2 a_{0} \lambda^{3} X}{2 a_{0} \lambda\left(a_{1}^{2}-a_{0}^{2} \lambda^{8}\right)},
$$

we can take this curve into the canonical form

$$
Y^{2} Z=X^{3}-3 a_{0}^{2} \lambda^{6} X^{2} Z-3 \lambda^{4} a_{0}^{2} \theta X Z^{2}-a_{0}^{2} \lambda^{2} \theta^{2} Z^{3}
$$

where $\theta=a_{1}^{2}-a_{0}^{2} \lambda^{8}$. If we write $X=\left(U+a_{0}^{2} \lambda^{6}\right) Z$ and $Y=W Z$, this becomes

$$
W^{2}=U^{3}-3 a_{0}^{2} a_{1}^{2} \lambda^{4} U-a_{0}^{2} a_{1}^{2} \lambda^{2}\left(a_{1}^{2}+\lambda^{8} a_{0}^{2}\right) .
$$

$\mathscr{F}_{3}$ is the only elliptic pencil on (20) that we shall use in the argument that follows. However, for possible applications elsewhere we record some useful information about the other pencils of low degree on (20).

There are two elliptic pencils of degree 4, which were described for the more general surface (6) in Section 3. There are four elliptic pencils of degree 6; a typical one, parametrized by $\alpha$, consists of the curves which are the intersections of the cubic surfaces

$$
\begin{aligned}
& a_{0}\left(X_{0}^{3}+X_{0}^{2} X_{2}+X_{0} X_{2}^{2}+X_{2}^{3}\right)+\alpha\left(X_{0} X_{3}-X_{1} X_{2}\right)\left(X_{1}-X_{3}\right)=0, \\
& a_{1}\left(X_{1}^{3}+X_{1}^{2} X_{3}+X_{1} X_{3}^{2}+X_{3}^{3}\right)-\alpha\left(X_{0} X_{3}-X_{1} X_{2}\right)\left(X_{0}-X_{2}\right)=0,
\end{aligned}
$$

residual to the three common lines $X_{0}=\kappa X_{2}, X_{1}=\kappa X_{3}$ where $\kappa=-1$ or $\kappa= \pm \sqrt{-1}$. This curve is a 2-covering of its Jacobian, and in canonical form it can be written

$$
Y^{2}=\alpha^{3}\left(a_{0}+a_{1} X^{4}\right)+a_{0} a_{1}\left(3 \alpha^{2}-a_{0} a_{1}\right) X^{2}
$$

where

$$
X=\frac{X_{1}+X_{3}}{X_{0}+X_{2}}, \quad Y=\frac{a_{1}\left(a_{0}+\alpha X^{2}\right)\left(X_{1}+X_{3}\right)}{X_{0}-X_{2}}
$$


We can recover the original variables by writing

$$
\begin{array}{ll}
X_{0}=Y+a_{1} X\left(a_{0}+\alpha X^{2}\right), & X_{1}=X Y-a_{0}\left(\alpha+a_{1} X^{2}\right), \\
X_{2}=Y-a_{1} X\left(a_{0}+\alpha X^{2}\right), & X_{3}=X Y+a_{0}\left(\alpha+a_{1} X^{2}\right) .
\end{array}
$$

The proof of Theorem 5 consists of a number of steps, each of which is described in a separate lemma. Throughout, $v$ will be the normalized 2-adic valuation on $\mathbb{Q}_{2}$ and $v(c)=\gamma$. In Lemmas 3 and $4, P_{0}=\left(x_{0}, x_{1}, x_{2}, x_{3}\right)$ will be a point of $V_{c}\left(\mathbb{Q}_{2}\right)$, with the $x_{i}$ odd; it then follows from the second equation of (21) that

$$
v(\lambda)+v\left(x_{0}+x_{2}\right)=\gamma+v\left(x_{1}+x_{3}\right) .
$$

The next two lemmas, taken together, provide for $V_{c}$ a quantitative version of Theorem 3. Let $\mathscr{F}_{3}$ be the fibration of degree 3 described by (21).

Lemma 3. Let $P_{0}$ be in $V_{c}(\mathbb{Q})$ with all $x_{i}$ odd, and let $F_{3}$ be the fibre of $\mathscr{F}_{3}$ through $P_{0}$.

(i) If $2 \|\left(x_{0}-x_{2}\right)$ and $2 \|\left(x_{1}-x_{3}\right)$, then $F_{3}(\mathbb{Q})$ is dense in that part of $F_{3}\left(\mathbb{Q}_{2}\right)$ in which the $X_{i}$ are odd with $2 \|\left(X_{1}-X_{3}\right)$ and $v\left(X_{0}+X_{2}\right) \geq v\left(x_{0}+x_{2}\right)$.

(ii) If $2 \|\left(x_{0}+x_{2}\right)$ and $2 \|\left(x_{1}-x_{3}\right)$, then $F_{3}(\mathbb{Q})$ is dense in that part of $F_{3}\left(\mathbb{Q}_{2}\right)$ in which the $X_{i}$ are odd.

Proof. Let $\mathscr{D}$ be that part of $F_{3}\left(\mathbb{Q}_{2}\right)$ in which we have to prove that $F_{3}(\mathbb{Q})$ is dense. In the notation of (22) and (23), let $x, y, z$ be the values of $X, Y, Z$ at $P_{0}$ and let $\lambda=\left(x_{0}-x_{2}\right) /\left(x_{1}-x_{3}\right)$ be the value of the parameter in (21) which defines $F_{3}$. We can take $a_{0}=1, a_{1}=c$.

In case (i), we have $v(\lambda)=0$; it now follows that $v\left(X_{1}+X_{3}\right)=v\left(2 a_{0} \lambda^{3} X\right)$ and $v(Z)>v(X)$. Thus $3 v(X / Z)=v(Y / X) \leq v(y / x)=3 v(x / z)<0$, where the equalities follow from (24). The conclusion now follows from Lemma 1.

In case (ii), we have $v(\lambda)=\gamma+\delta-1$ by (26), where $\delta=v\left(x_{1}+x_{3}\right) \geq 2$; so if we write $\xi=2^{-2 \gamma} X / Z$ and $\eta=2^{-3 \gamma} Y / Z$, the curve $F_{3}$ takes the form $\eta^{2}=\xi^{3}+\cdots$ with coefficients satisfying the conditions of Lemma 1 . At $P_{0}$ we have $v(x)=-\gamma, v(y)=0, v(z)=-3 \gamma$, where the last result again follows from $(26)$; so $v(\xi)=0$. Hence, by Lemma $1, F_{3}(\mathbb{Q})$ is dense in that part of $F_{3}\left(\mathbb{Q}_{2}\right)$ in which $v(\xi) \leq 0$. But in $\mathscr{D}$ we have

$$
v\left(X_{0}+X_{2}\right)=v\left(X_{1}+X_{3}\right)+\gamma-v(\lambda)=v\left(X_{1}+X_{3}\right)+1-\delta .
$$

Hence $v\left(X_{1}+X_{3}\right)<v\left(2 \lambda^{3} X\right)=v\left(X_{0}+X_{2}\right)+2 \gamma+3 \delta-3$, so that

$$
v(Z)=v\left(X_{1}+X_{3}\right)-v(\lambda)-2 \gamma-1=v\left(X_{0}+X_{2}\right)-3 \gamma-1
$$

whence $v(\xi)=v\left(X_{0}+X_{2}\right)-v(Z)-3 \gamma-1=0$. 
Lemma 4. Suppose there is a point $P_{0}$ of $V_{c}(\mathbb{Q})$ with all $x_{i}$ odd and $2^{2} \|\left(x_{1}+x_{3}\right)$. Let $\mathscr{D}_{1}$ be the part of $V_{c}\left(\mathbb{Q}_{2}\right)$ in which the $X_{i}$ are odd. Then $V_{c}(\mathbb{Q})$ is dense in $\mathscr{D}_{1}$.

Proof. Let $Q=\left(u_{0}, u_{1}, u_{2}, u_{3}\right)$ be a point of $\mathscr{D}_{1}$; after changing the signs of $u_{2}$ and/or $u_{3}$ if necessary, we can assume that $2 \|\left(u_{0}-u_{2}\right)$ and $2 \|\left(u_{1}-u_{3}\right)$, and we write $\lambda=\left(u_{0}-u_{2}\right) /\left(u_{1}-u_{3}\right)$. Similarly we can assume that $2 \|\left(x_{0}-x_{2}\right)$. Since $v(\lambda)=0$, the analogue for $Q$ of (26) shows that $v\left(u_{0}+u_{2}\right) \geq \gamma+2$. Now let $F_{3}^{\prime}$ be the fibre of $\mathscr{F}_{3}$ through $Q$. Write $\mu=\left(x_{0}+x_{2}\right) /\left(x_{1}-x_{3}\right)$ and let $F_{3}^{\prime \prime}$ be the intersection of $V_{c}$ with $X_{0}+X_{2}=\mu\left(X_{1}-X_{3}\right)$ residual to the line $X_{0}+X_{2}=X_{1}-X_{3}=0$; thus $P_{0}$ lies on $F_{3}^{\prime \prime}$.

Let $R=\left(y_{0}, y_{1}, y_{2}, y_{3}\right)$ be a point of $F_{3}^{\prime} \cap F_{3}^{\prime \prime}$. If we can ensure that the $y_{i}$ are odd elements of $\mathbb{Z}_{2}$ with $2\left\|\left(y_{0}-y_{2}\right), 2\right\|\left(y_{1}-y_{3}\right)$ and $v\left(u_{0}+u_{2}\right) \geq v\left(y_{0}+y_{2}\right)$, we can argue as follows. By Lemma 3(ii) with the sign of $X_{2}$ reversed, $F_{3}^{\prime \prime}(\mathbb{Q})$ is dense in that part of $F_{3}^{\prime \prime}\left(\mathbb{Q}_{2}\right)$ in which all the $X_{i}$ are odd integers; hence there is a point $R^{\prime}$ of $F_{3}^{\prime \prime}(\mathbb{Q})$ arbitrarily close to $R$. Let $F_{3}^{*}$ be the fibre of $\mathscr{F}_{3}$ through $R^{\prime}$, so that it is arbitrarily close to $F_{3}^{\prime}$. By Lemma 3(i) $F_{3}^{*}(\mathbb{Q})$ is dense in that part of $F_{3}^{*}\left(\mathbb{Q}_{2}\right)$ in which the $X_{i}$ are odd with $2 \|\left(X_{1}-X_{3}\right)$ and $v\left(X_{0}+X_{2}\right) \geq v\left(y_{0}+y_{2}\right)$, and in particular in that part of $F_{3}^{*}\left(\mathbb{Q}_{2}\right)$ which is close to $Q$. Hence there is a point of $V_{c}(\mathbb{Q})$ close to $Q$.

It remains to prove the assertions about $R$. The points of $F_{3}^{\prime} \cap F_{3}^{\prime \prime}$ are those points $\left(y_{0}, y_{1}, y_{2}, y_{3}\right)$ of $V_{c}$ at which

$$
y_{0}-y_{2}=\lambda\left(y_{1}-y_{3}\right), \quad y_{0}+y_{2}=\mu\left(y_{1}-y_{3}\right), \quad y_{1}-y_{3} \neq 0,
$$

where $v(\lambda)=0, v(\mu)=\gamma+\delta-1$. Write $y_{1}=y_{3}+2 \eta$; then $y_{0}=(\lambda+\mu) \eta$ and $y_{2}=(\mu-\lambda) \eta$, so that the equation for $R$ becomes

$$
\lambda \mu\left(\lambda^{2}+\mu^{2}\right) \eta^{3}+c\left(y_{3}+\eta\right)\left(y_{3}^{2}+2 y_{3} \eta+2 \eta^{2}\right)=0 .
$$

By Hensel's lemma, this has a solution in $\mathbb{Z}_{2}$ with $y_{3}, \eta$ odd; and for this solution $v\left(y_{3}+\eta\right)=\delta-1$ and thus $v\left(y_{1}+y_{3}\right)=\delta \leq v\left(u_{1}+u_{3}\right)$.

Suitable points $P_{0}$ exist for each of the three values of $c$. We can for example take $P_{0}$ to be $(849,653,-969,167)$ when $c=2,(189,677,-557,-657)$ when $c=4$, and $(1197,177,499,707)$ when $c=8$.

The next two lemmas again provide a quantitative version of Theorem 3 . In them we denote by $\mathscr{D}_{3}$ the part of $V_{c}\left(\mathbb{Q}_{2}\right)$ in which $X_{0}, X_{2}$ are odd and $X_{1}, X_{3}$ even, with just one of $X_{1}, X_{3}$ divisible by 4 ; and $P_{0}=\left(x_{0}, x_{1}, x_{2}, x_{3}\right)$ will be a point of $\mathscr{D}_{3}$ defined over $\mathbb{Q}$. As before let $x, y, z$ be the values of $X, Y, Z$ at $P_{0}$ and let $\lambda=\left(x_{0}-x_{2}\right) /\left(x_{1}-x_{3}\right)$ be the value of the parameter in (21) which defines $F_{3}$. We can take $a_{0}=1, a_{1}=c$.

Lemma 5. Let $F_{3}$ be the fibre of $\mathscr{F}_{3}$ through $P_{0}$, where $2 \|\left(x_{0}-x_{2}\right)$. Then $F_{3}(\mathbb{Q})$ is dense in $F_{3} \cap \mathscr{D}_{3}$. 
Proof. Since $v(\lambda)=0$, we have $v\left(X_{0}-X_{2}\right)=1$ at each point of $F_{3} \cap \mathscr{D}_{3}$. Thus at each such point $v\left(X_{0}+X_{2}\right)=\gamma+2$ and $v(X)=1, v(Y)=0, v(Z)=0$. In the notation of (25) we have $v(U)=v(W)=0$; and (25) satisfies the conditions of Lemma 1.

Lemma 6. Suppose that there is a point $P_{0}$ in $\mathscr{D}_{3}$ defined over $\mathbb{Q}$. Then $V_{c}(\mathbb{Q})$ is dense in $\mathscr{D}_{3}$.

Proof. Let $Q=\left(u_{0}, u_{1}, u_{2}, u_{3}\right)$ be a point of $\mathscr{D}_{3}$; after changing the sign of $x_{2}$ and/or $u_{2}$ if necessary, we can assume that $2 \|\left(x_{0}-x_{2}\right)$ and $2 \|\left(u_{0}-u_{2}\right)$. Write $\lambda=\left(u_{0}-u_{2}\right) /\left(u_{1}-u_{3}\right)$ and $\mu=\left(x_{0}-x_{2}\right) /\left(x_{1}+x_{3}\right)$. Let $F_{3}^{\prime}$ be the fibre of $\mathscr{F}_{3}$ through $Q$ and $F_{3}^{\prime \prime}$ the intersection of $V_{c}$ with $X_{0}-X_{2}=\mu\left(X_{1}+X_{3}\right)$ residual to the line $X_{0}-X_{2}=X_{1}+X_{3}=0$; thus $P_{0}$ lies on $F_{3}^{\prime \prime}$.

Let $R=\left(y_{0}, y_{1}, y_{2}, y_{3}\right)$ be a point of $F_{3}^{\prime} \cap F_{3}^{\prime \prime}$. If we write $y_{0}+y_{3}=\chi\left(y_{0}-y_{2}\right)$ and use $y_{0}-y_{2}=\lambda\left(y_{1}-y_{3}\right)=\mu\left(y_{1}+y_{3}\right)$, we find that $\chi$ satisfies

$$
\chi\left(\chi^{2}+1\right)=c\left(\lambda^{2}+\mu^{2}\right)(\lambda \mu)^{-3} .
$$

Since $v(\lambda)=v(\mu)=0$, this equation is satisfied by one value of $\chi$ in $\mathbb{Q}_{2}$ with $v(\chi)=\gamma+1$, and the corresponding $R$ is in $\mathscr{D}_{3}$. The lemma now follows by the same argument as in the second paragraph of the proof of Lemma 4.

Suitable points $P_{0}$ exist for each of the three values of $c$. We can for example take $P_{0}$ to be $(489,684,-577,662)$ for $c=2,(61,168,-237,58)$ for $c=4$, and $(257,22,223,124)$ for $c=8$.

Henceforth, for any integer $\beta>0$ let $\mathscr{D}_{4}^{\beta}$ consist of those points of $V_{c}\left(\mathbb{Q}_{2}\right)$ with $X_{0}, X_{2}$ odd and $v\left(X_{1}\right)=v\left(X_{3}\right)=\beta$ and let $\mathscr{D}_{5}^{\beta}$ consist of those points of $V_{c}\left(\mathbb{Q}_{2}\right)$ with $X_{0}, X_{2}$ odd and $v\left(X_{1}\right)>v\left(X_{3}\right)=\beta$ or $v\left(X_{3}\right)>v\left(X_{1}\right)=\beta$. Thus $\mathscr{D}_{5}^{1}$ is what we have previously called $\mathscr{D}_{3}$. To complete the proof of Theorem 5 we need to prove that $V_{c}(\mathbb{Q})$ is dense in each $\mathscr{D}_{4}^{\beta}$ with $\beta>0$ and each $\mathscr{D}_{5}^{\beta}$ with $\beta>1$.

Lemma 7. If $V_{c}(\mathbb{Q})$ is dense in $\mathscr{D}_{1}$ then it is dense in $\mathscr{D}_{5}^{\beta}$ for each $\beta>1$.

Proof. Let $Q=\left(u_{0}, u_{1}, u_{2}, u_{3}\right)$ be a point of $\mathscr{D}_{5}^{\beta}$ where $\beta>1$; after changing the sign of $u_{2}$ if necessary, we can assume that $2 \|\left(u_{0}-u_{2}\right)$ and therefore $v\left(u_{0}+u_{2}\right)=4 \beta-2$. Let $\lambda=\left(u_{0}-u_{2}\right) /\left(u_{1}-u_{3}\right)$, so that $v(\lambda)=1-\beta$. At $Q$ we have $v(X)=4 \beta-3$, $v(Y)=\beta-1$ and therefore $v(Z)=10(\beta-1)$; hence also $v(U)=6(1-\beta)$, $v(W)=9(1-\beta)$. If we write $\xi=2^{4(\beta-1)} U, \eta=2^{6(\beta-1)} W$ in (25), then the equation between $\xi$ and $\eta$ satisfies the conditions of Lemma 1. In the notation of that lemma, let $R=\left(y_{0}, y_{1}, y_{2}, y_{3}\right)$ be the point of $E^{\prime}$ which satisfies $\zeta(R)=2^{1-\beta} \zeta(Q) ; R$ exists because $v(\zeta(Q))=\beta-1$. Thus $v(\xi / \eta)=0$ at $R$, and so $v(\xi)=v(\eta)=0$ since $R$ is in $E^{\prime}$. Moreover if we choose the representation of $R$ so that $v\left(y_{0}-y_{2}\right)=1$, we have $v\left(y_{1}-y_{3}\right)=1-v(\lambda)=\beta$; so $v(Y)=\beta-1, v(Z)=7(\beta-1), v(X)=\beta-1$, 
$v\left(y_{0}+y_{2}\right)=\beta+\gamma>1$ at $R$. The results for $y_{0} \pm y_{2}$ show that $y_{0}$ and $y_{2}$ are odd integers. If we assume that $v\left(y_{1}+y_{3}\right) \leq 0$, we find that

$$
\beta+\gamma+2=v\left(y_{0}^{4}-y_{2}^{4}\right)=\gamma+v\left(y_{1}^{4}-y_{3}^{4}\right) \leq \beta+\gamma-1,
$$

and if instead we assume that $v\left(y_{1}+y_{3}\right) \geq 2$, we find that

$$
\beta+\gamma+2=v\left(y_{0}^{4}-y_{2}^{4}\right)=\gamma+v\left(y_{1}^{4}-y_{3}^{4}\right) \geq \beta+\gamma+4,
$$

both of which are absurd. So we must have $v\left(y_{1}+y_{3}\right)=1$, whence $R$ is in $\mathscr{D}_{1}$. Hence there is a point $R^{\prime}$ in $V_{c}(\mathbb{Q})$ arbitrarily close to $R$. Let $Q^{\prime}=\left[2^{\beta-1}\right] R^{\prime}$, where the operation is to be carried out on the fibre of $\mathscr{F}_{3}$ through $R^{\prime}$; then $Q^{\prime}$ is in $V_{c}(\mathbb{Q})$ and arbitrarily close to $Q$.

Lemma 8. If $V_{c}(\mathbb{Q})$ is dense in $\mathscr{D}_{1}$ then it is dense in $\mathscr{D}_{4}^{\beta}$ for each $\beta>0$.

Proof. Let $Q=\left(u_{0}, u_{1}, u_{2}, u_{3}\right)$ be a point of $\mathscr{D}_{4}^{\beta}$ where $\beta>0$; after changing the signs of $u_{2}$ and/or $u_{3}$ if necessary, we can assume that $v\left(u_{0}-u_{2}\right)=1$ and $v\left(u_{1}-u_{3}\right)=\beta+1$. Thus $v\left(u_{1}+u_{3}\right)=\beta+\delta$ for some $\delta>1$, whence $v\left(u_{0}+u_{2}\right)=$ $4 \beta+\gamma+\delta$. Let $\lambda=\left(u_{0}-u_{2}\right) /\left(u_{1}-u_{3}\right)$, so that $v(\lambda)=-\beta$. At $Q$ we have $v(X)=4 \beta+\delta-1, v(Y)=\beta$ and therefore $v(Z)>10 \beta+\delta-1$ and it now follows from (24) that $v(Z)=10 \beta+3 \delta-3$. Hence $v(U)=-6 \beta-2 \delta+2$ and $v(W)=-9 \beta-3 \delta+3$. If we write $\xi=2^{4 \beta} U, \eta=2^{6 \beta} W$ in (25) then the equation between $\xi$ and $\eta$ satisfies the conditions of Lemma 1. In the notation of that lemma, let $R=\left(y_{0}, y_{1}, y_{2}, y_{3}\right)$ be the point of $E^{\prime}$ which satisfies $\zeta(R)=2^{1-\beta-\delta} \zeta(Q) ; R$ exists because $v(\zeta(Q))=\beta+\delta-1$. Thus $v(\xi / \eta)=0$ at $R$, and so $v(\xi)=v(\eta)=0$ since $R$ is in $E^{\prime}$. Moreover if we choose the representation of $R$ so that $v\left(y_{0}-y_{2}\right)=1$ we have $v\left(y_{1}-y_{3}\right)=1-v(\lambda)=1+\beta$; so $v(Y)=\beta, v(Z)=7 \beta, v(X)=\beta$, $v\left(y_{0}+y_{2}\right)=\beta+\gamma+1>2$ at $R$. The results for $y_{0} \pm y_{2}$ show that $y_{0}$ and $y_{2}$ are odd integers. If we assume that $v\left(y_{1}+y_{3}\right) \leq 0$, we find that

$$
\beta+\gamma+3=v\left(y_{0}^{4}-y_{2}^{4}\right)=\gamma+v\left(y_{1}^{4}-y_{3}^{4}\right) \leq \beta+\gamma,
$$

and if instead we assume that $v\left(y_{1}+y_{3}\right) \geq 2$, we find that

$$
\beta+\gamma+3=v\left(y_{0}^{4}-y_{2}^{4}\right)=\gamma+v\left(y_{1}^{4}-y_{3}^{4}\right) \geq \beta+\gamma+5,
$$

both of which are absurd. So we must have $v\left(y_{1}+y_{3}\right)=1$, whence $R$ is in $\mathscr{D}_{1}$. Hence there is a point $R^{\prime}$ in $V_{c}(\mathbb{Q})$ arbitrarily close to $R$. Let $Q^{\prime}=\left[2^{\beta+\delta-1}\right] R^{\prime}$, where the operation is to be carried out on the fibre of $\mathscr{F}_{3}$ through $R^{\prime}$; then $Q^{\prime}$ is in $V_{c}(\mathbb{Q})$ and arbitrarily close to $Q$.

Proof of Theorem 5. $V_{c}(\mathbb{Q})$ is dense in $\mathscr{D}_{1}$ by Lemma 4, and dense in $\mathscr{D}_{3}$ by Lemma 6. It is dense in the rest of that part of $V_{c}\left(\mathbb{Q}_{2}\right)$ in which the $X_{i}$ are integers with $X_{0}, X_{2}$ odd, by Lemmas 7 and 8 . This is all we need. 
Very little extra effort is needed to take account also of the infinite place.

Corollary. The image of $V_{c}(\mathbb{Q})$ is dense in $V_{c}\left(\mathbb{Q}_{2}\right) \times V_{c}(\mathbb{R})$.

The argument in the last paragraph of the proof of Theorem 3 still works, and it shows that the image of $V_{c}(\mathbb{Q})$ is dense in at least one of the $V_{c}\left(\mathbb{Q}_{2}\right) \times \mathscr{R}$, where $\mathscr{R}$ is one of the connected components of $V_{c}(\mathbb{R}) \backslash(Z \cup X)$. As in the proof of Theorem 4, each of these connected components is given by fixing the signs of the $X_{i} / X_{j}$, and because we can change the sign of any $x_{i}$ the corollary follows.

\section{References}

[Bogomolov and Tschinkel 1998] F. A. Bogomolov and Y. Tschinkel, "Density of rational points on Enriques surfaces”, Math. Res. Lett. 5:5 (1998), 623-628. MR 99m:14040 Zbl 0957.14016

[Bright 2002] M. Bright, Computations on diagonal quartic surfaces, Ph.D. thesis, University of Cambridge, 2002, Available at http://www.boojum.org.uk/maths/quartic-surfaces/thesis.pdf.

[Colliot-Thélène and Skorobogatov 2013] J.-L. Colliot-Thélène and A. N. Skorobogatov, "Good reduction of the Brauer-Manin obstruction", Trans. Amer. Math. Soc. 365:2 (2013), 579-590. MR 2995366

[Ieronymou et al. 2011] E. Ieronymou, A. N. Skorobogatov, and Y. G. Zarhin, "On the Brauer group of diagonal quartic surfaces", J. Lond. Math. Soc. (2) 83:3 (2011), 659-672. MR 2012e:14046 Zbl 1239.14013

[Logan et al. 2010] A. Logan, D. McKinnon, and R. van Luijk, "Density of rational points on diagonal quartic surfaces”, Algebra Number Theory 4:1 (2010), 1-20. MR 2011a:11126 Zbl 1206.11082

[Pannekoek 2012] R. Pannekoek, “On $p$-torsion of $p$-adic elliptic curves with additive reduction", preprint, 2012. arXiv 1211.5833

[Silverman 1986] J. H. Silverman, The arithmetic of elliptic curves, Graduate Texts in Mathematics 106, Springer, New York, 1986. MR 87g:11070 Zbl 0585.14026

[Swinnerton-Dyer 1968] H. P. F. Swinnerton-Dyer, “ $A^{4}+B^{4}=C^{4}+D^{4}$ revisited”, J. London Math. Soc. 43 (1968), 149-151. MR 37 \#2685 Zbl 0206.33703

[Swinnerton-Dyer 2000] P. Swinnerton-Dyer, “Arithmetic of diagonal quartic surfaces, II", Proc. London Math. Soc. (3) 80:3 (2000), 513-544. MR 2001d:11069 Zbl 1066.11029

[Swinnerton-Dyer 2001] P. Swinnerton-Dyer, "Weak approximation and $R$-equivalence on cubic surfaces", pp. 357-404 in Rational points on algebraic varieties, edited by E. Peyre and Y. Tschinkel, Progr. Math. 199, Birkhäuser, Basel, 2001. MR 2003c:11070 Zbl 1079.11035

Communicated by Jean-Louis Colliot-Thélène

Received 2010-12-16 Revised 2012-10-01 Accepted 2012-12-10

H.P.F.Swinnerton-Dyer@dpmms.cam.ac.uk

Department of Pure Mathematics and Mathematical Statistics, University of Cambridge, Cambridge, CB3 OWB, United Kingdom 


\section{Algebra \& Number Theory}

msp.org/ant

\section{EDITORS}

MANAGING EDITOR

Bjorn Poonen

Massachusetts Institute of Technology

Cambridge, USA

\author{
EDITORIAL BOARD CHAIR \\ David Eisenbud \\ University of California \\ Berkeley, USA
}

\section{BOARD OF EDITORS}

Georgia Benkart

Dave Benson

Richard E. Borcherds

John H. Coates

J-L. Colliot-Thélène

Brian D. Conrad

Hélène Esnault

Hubert Flenner

Edward Frenkel

Andrew Granville

Joseph Gubeladze

Ehud Hrushovski

Craig Huneke

Mikhail Kapranov

Yujiro Kawamata

János Kollár

Yuri Manin

Barry Mazur

Philippe Michel
University of Wisconsin, Madison, USA

University of Aberdeen, Scotland

University of California, Berkeley, USA

University of Cambridge, UK

CNRS, Université Paris-Sud, France

University of Michigan, USA

Freie Universität Berlin, Germany

Ruhr-Universität, Germany

University of California, Berkeley, USA

Université de Montréal, Canada

San Francisco State University, USA

Hebrew University, Israel

University of Virginia, USA

Yale University, USA

University of Tokyo, Japan

Princeton University, USA

Northwestern University, USA

Harvard University, USA

École Polytechnique Fédérale de Lausanne
Susan Montgomery

Shigefumi Mori

Raman Parimala

Jonathan Pila

Victor Reiner

Karl Rubin

Peter Sarnak

Joseph H. Silverman

Michael Singer

Vasudevan Srinivas

J. Toby Stafford

Bernd Sturmfels

Richard Taylor

Ravi Vakil

Michel van den Bergh

Marie-France Vignéras

Kei-Ichi Watanabe

Efim Zelmanov
University of Southern California, USA

RIMS, Kyoto University, Japan

Emory University, USA

University of Oxford, UK

University of Minnesota, USA

University of California, Irvine, USA

Princeton University, USA

Brown University, USA

North Carolina State University, USA

Tata Inst. of Fund. Research, India

University of Michigan, USA

University of California, Berkeley, USA

Harvard University, USA

Stanford University, USA

Hasselt University, Belgium

Université Paris VII, France

Nihon University, Japan

University of California, San Diego, USA

\section{PRODUCTION}

production@msp.org

Silvio Levy, Scientific Editor

See inside back cover or msp.org/ant for submission instructions.

The subscription price for 2013 is US \$200/year for the electronic version, and \$350/year ( $\$ 40$, if shipping outside the US) for print and electronic. Subscriptions, requests for back issues and changes of subscribers address should be sent to MSP.

Algebra \& Number Theory (ISSN 1944-7833 electronic, 1937-0652 printed) at Mathematical Sciences Publishers, 798 Evans Hall \#3840, c/o University of California, Berkeley, CA 94720-3840 is published continuously online. Periodical rate postage paid at Berkeley, CA 94704, and additional mailing offices.

ANT peer review and production are managed by EditFLOW ${ }^{\circledR}$ from Mathematical Sciences Publishers.

\section{PUBLISHED BY}

- mathematical sciences publishers

nonprofit scientific publishing

http://msp.org/

(C) 2013 Mathematical Sciences Publishers 


\section{Algebra \& Number Theory}

$\begin{array}{lll}\text { Volume } 7 & \text { No. } 4 \quad 2013\end{array}$

Explicit Chabauty over number fields

SAMIR SIKSEK

Moduli spaces for point modules on naïve blowups

Thomas A. Nevins and Susan J. Sierra

Density of rational points on certain surfaces

Sir Peter SWInNerton-Dyer

HENRIK RUSSELL

Chai's conjecture and Fubini properties of dimensional motivic integration

RAF Cluckers, François LoESER and JohanNes NiCAISE

SHUNSUKE TAKAGI

Finitely presented exponential fields

JONATHAN KIRBY

On a problem of Arnold: The average multiplicative order of a given integer

PÄr Kurlberg and CARL POMERANCE

An analogue of Sturm's theorem for Hilbert modular forms 